\title{
Calculation of Free Volume in Computer Generated Grain Boundaries
}

\author{
S. VAn Petegem ${ }^{a}$ And J. Kuriplach ${ }^{b}$ \\ ${ }^{a}$ NUM/ASQ, Paul Scherrer Institut, 5232 Villigen PSI, Switzerland \\ ${ }^{b}$ Department of Low Temperature Physics, Charles University \\ V Holešovičkách 2, 18000 Prague, Czech Republic
}

We investigate the positron lifetime distribution in grain boundaries originating from computer generated nanocrystalline configurations. We give a brief overview of the computational methods and we discuss the influence of thermal vibrations and grain size on the obtained positron lifetime distributions.

PACS numbers: 78.70.Bj, 61.46.+w, 61.72.Mm, 68.35.Dv

\section{Introduction}

The reduction of the grain size down to the $\mathrm{nm}$ regime has opened new and fascinating avenues for research in several aspects of materials science, including mechanical properties. In recent years large efforts, both experimental and computational, have been made to understand the deformation mechanisms at the nanoscale. Computer simulations suggest both grain boundary accommodation mechanisms and intra granular slip involving dislocation emission and absorption at grain boundaries, as possible deformation mechanisms. In these simulations the structure of the grain boundaries plays a crucial role.

In the past 15 years positron annihilation lifetime spectroscopy has been used extensively to investigate the defect structure at grain boundaries in nanocrystalline (nc) materials. However, grain boundaries are often composed of very complex defects structures that, in principle, cannot be described by one or two single positron lifetimes components. Recently we have developed a method to calculate the positron signal in very large simulated nanostructures, which enables us to gain a better understanding of the free volume distribution in grain boundaries [1-4]. 
In previous work we have compared the positron lifetime distribution in grain boundaries synthesized by different methods [1]. In this paper we focus on the positron lifetime characteristics of grain boundaries synthesized using a Voronoi construction. We investigate the influence of thermal vibrations and grain size on the positron lifetime distribution.

\section{Computational details}

\subsection{Computer simulated samples}

The nc configurations used in the paper have been studied extensively in the past (see e.g. Refs. [5-8]). They are synthesized using a Voronoi construction. The starting configuration is an empty simulation cell with fully $3 \mathrm{D}$ periodic boundary conditions in which randomly a number of positions is chosen. From each position, an fcc lattice is constructed geometrically. At a point where atoms from one grain center are closer to the center of another grain, construction is halted. Eventually construction will cease throughout the entire sample, resulting in a three-dimensional granular structure according to the Voronoi construction [9]. At this stage atom pairs, each atom originating from a different crystallite, are inspected and where there is a nearest neighbor distance of less than $2 \AA$, one atom is removed. Molecular statics is then performed to relax any local high potential energy configuration that may exist, followed by NPT MD (molecular dynamics (MD) with constant pressure, temperature, and number of particles) at room temperature to further relax and equilibrate the structure. The constant pressure MD is performed using the Parrinello-Rahman Lagrangian [10] with orthorhombic

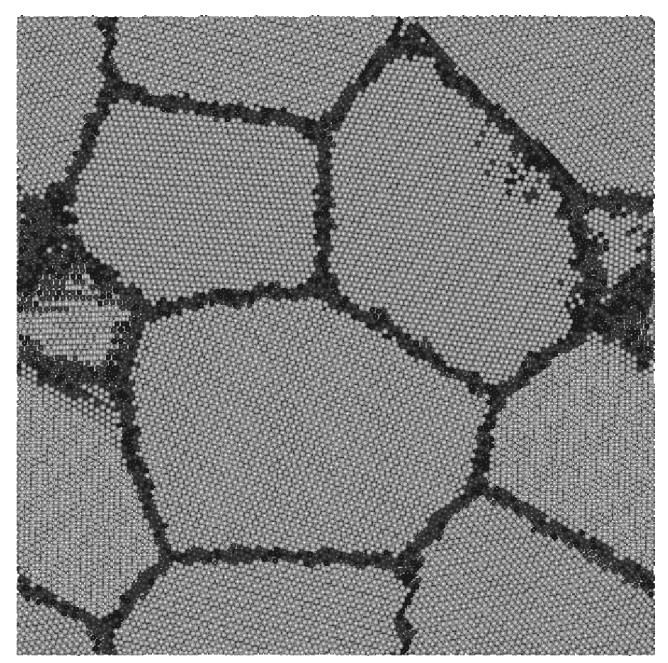

Fig. 1. Typical cross-section of Ni12 showing the grains (grey colored atoms) separated by the grain boundaries (dark colored atoms). 
simulation cell geometry conditions. For this work we have studied three samples consisting of 15 grains with a grain size of 5,12 , and $20 \mathrm{~nm}$, the only difference being the distance between the grain centers. They are referred to as, respectively, $\mathrm{Ni} 5$, Ni12, and Ni20. Characteristic of these types of nc configurations are the full density, defect-free grains and grain boundaries with a large degree of order. Figure 1 shows a typical cross-section of Ni12. To identify the grain boundary (GB) network structure a medium range environmental analysis is performed for each atom, where a local crystallinity class is assigned to each atom [11]. This allows us to separate fcc, hcp, and other coordinated atoms, which makes the visualization of the grain boundary structure straightforward, as shown in Fig 1.

\subsection{Positron lifetime calculations}

The positron annihilation lifetime calculations were performed using the standard atomic superposition (ATSUP) method $[12,13]$. It is a non-selfconsistent technique in which the electron densities and Coulomb potential are superimposed numerically on a three-dimensional mesh according to the atomic structure of the system. The total positron potential is constructed as a sum of the electron Coulomb potential originating from the superposition of atomic potentials and the correlation potential, which originates from the interaction between the positron and the electrons. Then, the Schrödinger equation for positrons is solved using a conjugate gradient method [14] on the nodes of a three-dimensional mesh, resulting in the positron wave function and energy eigenvalue. Finally the positron lifetime is calculated by the following formula:

$$
1 / \tau=\pi r_{\mathrm{e}}^{2} c \int n_{-}(\boldsymbol{r}) n_{+}(\boldsymbol{r}) \gamma\left(n_{-}(\boldsymbol{r})\right) \mathrm{d} \boldsymbol{r},
$$

where $r_{\mathrm{e}}$ is the classical electron radius, $c$ is the speed of light, $n_{+}$and $n_{-}$are, respectively, the positron and electron density, and $\gamma$ is the so-called enhancement factor describing the pile-up of electrons around the positrons. In our calculations the parametrization of $\gamma$ and the correlation potential according to Ref. [15] was employed.

In the calculations of positron characteristics in complex structures such as grain boundaries the calculation boxes are generally non-periodic and contain more than one positron trap. Therefore the standard ATSUP method has been adapted to overcome these problems. First the electron densities and electron Coulomb potentials are constructed as usual but for the further considerations a smaller (inner) part of the cut is taken into account to avoid an influence of the "false" imposed periodicity. Furthermore the positron potential at the side of the box is appropriately adjusted to omit artificial shallow positron traps at these sides. After these adjustments the cut can be considered periodic when solving the Schrödinger equation. To consider more than one positron trap in the grain boundary, the ATSUP method was extended so that it is possible to look for more positron states [14]. This procedure is described in more detail in Refs. [2-4]. 
The nc configurations contain up to 5 million atoms, which is too large for the positron lifetime calculations. Therefore smaller cuts have to be made to create the input files for the ATSUP program. First a very fine mesh with a typical mesh spacing of $0.2 \AA$ is created over the whole simulated sample. For every mesh point the distance to the nearest atoms is calculated. If the distance to the nearest atom is larger than $2 \AA$ this mesh point is marked as belonging to a free volume. Then neighboring "free volume mesh points" are concatenated into one free volume. Finally appropriate input files are created by selecting a box with a certain minimum and maximum size around the free volumes sites.

\section{Results}

\subsection{Thermal vibrations}

When calculating the positron lifetime distribution in any configuration at non-zero temperature thermal vibrations have to be taken into account. This is especially true for atoms located at grain boundaries. We demonstrate this for Ni12 in a random instantaneous configuration and a configuration that was averaged for 1 ps. Within this time frame the diffusion of atoms is negligible. Figure 2 displays the distribution of the deviation of atoms from their averaged position. Clearly the grain boundary atoms show much larger displacements. This is in agreement with the results obtained in Ref. [16] where the mean square displacement (MSD) along one direction is calculated from the vibrational density of states. It is shown that grain boundary atoms have an MSD twice as large compared to atoms in the grain interiors. Obviously these large displacements have a profound influence on the free volume distribution in the grain boundaries.

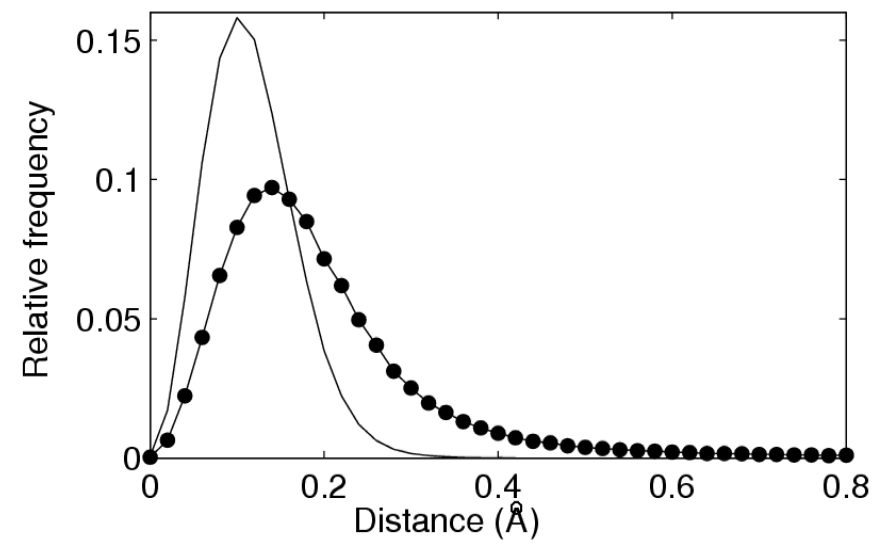

Fig. 2. Deviation of fcc atoms (full line) and grain boundary atoms (circles) from their averaged position. 


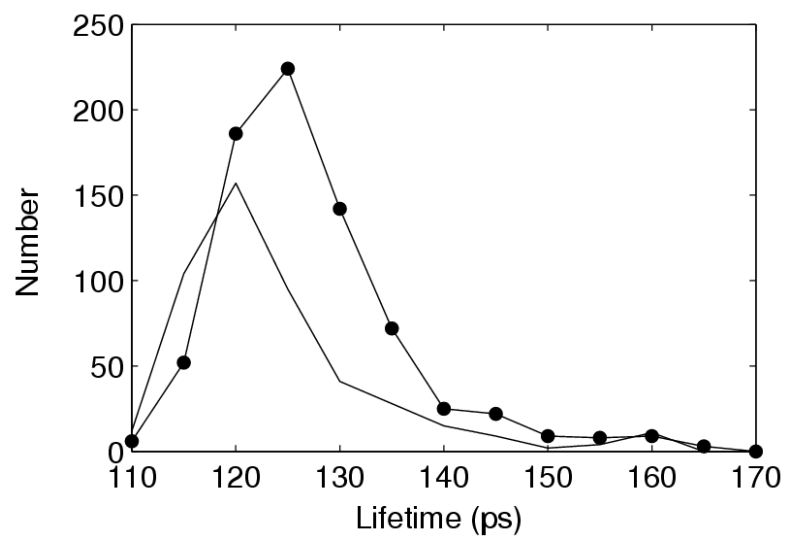

Fig. 3. Positron lifetime distribution in Ni12 for an instantaneous (circles) and an averaged configuration (full line).

Figure 3 shows the calculated positron lifetime distribution of the instantaneous and averaged configuration. In the instantaneous configuration more free volume sites have been detected. Furthermore the positron lifetime distribution is slightly shifted towards larger positron lifetimes. This is due to the fact that we only investigate free volume sites with a radius larger than $2 \AA$. Due to the thermal vibration of the atoms the size of the free volume sites can drop below, or increase above this limit. Experimental positron lifetimes are at least 3 orders of magnitude longer than the time scale of thermal vibrations. Therefore the positron lifetime reflects the free volume created by the averaged positions of the atoms.

\subsection{Grain size effect}

Another important issue is the grain size dependence of the grain boundary structure. In coarse-grained polycrystalline metals, plasticity is carried by dislocations generated by sources within the grains. But there is a critical length scale below which a dislocation source can no longer operate because the stress to bow out a dislocation approaches the theoretical shear stress [17]. To study this phenomenon the deformation behavior of Ni5 and Ni12 has been investigated extensively in the past (see e.g. Ref. $[5,7]$ ). In order to investigate whether the observed features are solely due to a grain size effect and not to a change of the grain boundary structure, we have studied the free volume distribution in the grain boundaries as a function of grain size. Figure 4 displays the positron lifetime distribution for Ni5, Ni12, and Ni20. As Ni5 contains only 44 free volume sites the calculations were also performed on a sample containing 125 grains with a mean grain size of $5 \mathrm{~nm}$. The resulting positron lifetime distribution coincides perfectly with the one of Ni5 (not shown in the figure for clarity), indicating that the results for Ni5 are reliable. From Fig. 4 it is clear that with decreasing grain size there is a tendency towards larger free volumes. 


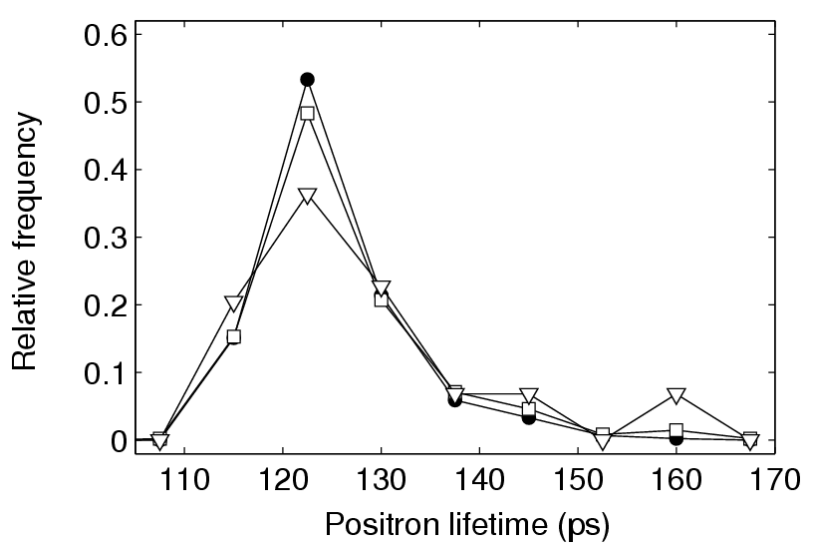

Fig. 4. Positron lifetime distribution for Ni20 (close circles), Ni12 (open squares), and Ni5 (open triangles).

We have investigate several possible reasons for this behavior. One of the main differences between the samples is the fraction of triple junctions. Therefore we have investigated separately the free volume distribution in the grain boundaries and in the triple junctions. However, no significant differences could be observed. Another reason might be that $\mathrm{Ni} 5$ is under a larger stress than Ni12 and Ni20, due to the small grain size. This could have an influence on the grain boundary microstructure. In Ref. [16] the local stress distribution for fcc atoms is calculated. It is shown that for $\mathrm{Ni} 5$ the width of the local stress distribution is clearly larger compared to Ni12. However, when we calculate the local stress distribution for non-fcc atoms, the distributions are nearly the same. Therefore we do not believe that this is the cause for the observed differences in the positron lifetime distribution.

\section{Conclusion}

We have investigated the positron lifetime distribution in grain boundaries synthesized using a Voronoi construction. We show that thermal vibrations have a rather large influence on the positron response in grain boundaries. Furthermore there is an indication that the positron lifetime distribution slightly depends on the grain size. The exact reason for this feature is currently under investigation.

\section{Acknowledgment}

We are grateful to H. Van Swygenhoven and P.M. Derlet for providing us with their nanocrystalline configurations. We also thank M.J. Puska and T. Korhonen for the ATSUP positron code. 


\section{References}

[1] S. Van Petegem, J. Kuriplach, H. Van Swygenhoven, R. Meyer, D. Segers, C. Dauwe, Mater. Sci. Forum 445-446, 204 (2004).

[2] J. Kuriplach, S. Van Petegem, M. Hou, E.E. Zhurkin, H. Van Swygenhoven, F. Dalla Torre, G. Van Tendeloo, M. Yandouzi, D. Schryvers, D. Segers, A.L. Morales, S. Ettaoussi, C. Dauwe, Mat. Sci. For 363-365, 94 (2001).

[3] J. Kuriplach, S. Van Petegem, D. Segers, C. Dauwe, M. Hou, E.E. Zhurkin, H. Van Swygenhoven, A.L. Morales, in: Mater. Res. Soc. Symp. Proc. Structure and Mechanical Properties of Nanophase Materatials - Theory and Computer Simulations vs. Experiment, Eds. D. Farkas, H. Kung, M. Mayo, H. Van Swygenhoven, J. Weertman, Vol. 634, Materials Research Society, Boston 2001, p. B.3.8.1.

[4] J. Kuriplach, Appl. Surf. Sci. 194, 61 (2002).

[5] P.M. Derlet, H. Van Swygenhoven, Phys. Rev. B 67, 014202 (2003).

[6] A. Hasnaoui, H. Van Swygenhoven, P.M. Derlet, Acta Mater. 50, 3927 (2002).

[7] H. Van Swygenhoven, P.M. Derlet, A. Hasnaoui, Phys. Rev. B 66, 024101 (2002).

[8] H. Van Swygenhoven, P.M. Derlet, Phys. Rev. B 64, 224105 (2001).

[9] G.Z. Voronoi, Reine Angew. Math. 134, 199 (1908).

[10] M. Parrinello, A. Rahman, J. Appl. Phys. 52, 7182 (1981).

[11] J.D. Honeycutt, H.C. Andersen, J. Phys. Chem. 91, 4950 (1987).

[12] M.J. Puska, R.M. Nieminen, J. Phys. F 13, 333 (1983).

[13] A.P. Seitsonen, M.J. Puska, M.J. Niemenen, Phys. Rev. B 51, 14057 (1995).

[14] M.P. Teter, M.C. Payne, D.C. Alan, Phys. Rev. B 40, 1225 (1989).

[15] E. Boroński, R.M. Nieminen Phys. Rev. B 34, 3820 (1986).

[16] P.M. Derlet, S. Van Petegem, H. Van Swygenhoven, Phys. Rev. B 71, 024114 (2005).

[17] M. Legros, B.R. Elliott, M.N. Rittner, J.R. Weertman, K.J. Hemker, Philos. Magn. A 80, 1017 (2000). 